\title{
Effectiveness of Time Diversity against Multi-Frequency Disturbances Under Plane Wave Conditions
}

\author{
Hassan Tirmizi \\ Dept. of Electrical Engineering \\ KU Leuven, Bruges Campus \\ Bruges, Belgium \\ hassan.tirmizi@kuleuven.be
}

\author{
Jonas Lannoo \\ Dept. of Electrical Engineering \\ KU Leuven, Bruges Campus \\ Bruges, Belgium \\ jonas.lannoo@kuleuven.be
}

\author{
Dries Vanoost \\ Dept. of Electrical Engineering \\ KU Leuven, Bruges Campus \\ Bruges, Belgium \\ dries.vanoost@kuleuven.be
}

\author{
Guy A. E. Vandenbosch \\ Dept. of Electrical Engineering \\ KU Leuven, Arenberg Campus \\ Leuven, Belgium \\ guy.vandenbosch@kuleuven.be
}

\author{
Davy Pissoort \\ Dept. of Electrical Engineering \\ KU Leuven, Bruges Campus \\ Bruges, Belgium \\ davy.pissoort@kuleuven.be
}

\begin{abstract}
In this paper, time diversity is used as a technique to achieve EM resilience in a redundant communication channel that is subjected to multi-frequency electromagnetic disturbances. The geometry under study is subjected to strong incident fields consisting of random angles of incidence and polarizations. Time diversity is used as a measure to achieve fault tolerance in the system. The effects of a multi-harmonic Electromagnetic Interference (EMI) on the functional transmission signal inside a redundant three-channel system with time diversity is compared to the base case with no time diversity.

The study shows that time diversity proves to be quite an effective measure to incorporate fault tolerance in redundant systems which are interfered by multi-frequency disturbances. The results reflect a clear improvement in performance in terms of the Bit Error Rates (BERs). Furthermore, results show that time diversity when suitably employed for a particular EMI disturbance can lead to optimal improvement in performance.
\end{abstract}

Index Terms-Electromagnetic Interference (EMI), EMI Resilience, Statistical Electromagnetics, Electromagnetic Compatibilty (EMC), Autonomous Systems

\section{INTRODUCTION}

The technological development that mankind has witnessed over the last quarter of a century has been quite impressive and awe-inspiring. Modern engineering marvels such as selfdriving cars and autonomous robots are highly intricate in nature wherein a number of enabling technologies are simultaneously at work. Besides, there has been a great emphasis on making highly automated systems with minimal levels

The research leading to these results has received funding from the European Union's Horizon 2020 research and innovation programme under the Marie Skłodowska-Curie Grant Agreement No 812.788 (MSCA-ETN SAS). This publication reflects only the authors' view, exempting the European Union from any liability.

978-1-7821-5579-1/20/\$31.00 @2020 IEEE of human intervention. However, dependability becomes a major concern whenever we talk about highly automated or autonomous systems.

In these highly complex, mission-critical systems, the potential hazards that can occur due to EMI can hardly be overestimated. Therefore, it is imperative to apply a risk-based approach to EMC as the current rule and test based approach is insufficient to provide a true estimate of all reasonably foreseeable failures that can arise due to EMI [1]. The novel discipline of EMI resilience aims to incorporate various risk reduction measures within the design itself so as to limit the safety risks due to EMI to a tolerable level [3], [4]. The IEEE EMC Society's standard development project P1848 [4] enlists a set of EMI resilience techniques and measures, which can help in realizing systems which are inherently more robust to EMI. Since EMI leads to systematic, common-cause failures inside a system, many of the techniques from the discipline of functional safety for embedding fault tolerance are proposed for a more robust EMC design [4]. One of those techniques for improving the resilience of safety-critical systems to EMI is to combine redundancy with some form of diversity in order to realize an EM-diverse system. Various successful implementations of EM-diverse systems, i.e. time diversity [5], frequency diversity [11], spatial diversity [6] and different matching schemes [7] have been reported in literature for single frequency disturbances. The research in [5] indicated that time diversity when optimised for the EMI disturbance's frequency gives the best performance.

This paper extends the concept of time diversity further by using it as a countermeasure against multi-frequency disturbances. Time diversity results from [5] show a considerable improvement in performance in terms of the bit error rate when implemented optimally for a single frequency excitation. 
However, extending the concept of time diversity to multifrequency disturbances leads to a more complex range of scenarios wherein the optimal time diversity is not evident right away.

The remainder of this paper is organized in a way that in Section II, the simulation model and the concept of time diversity are explained. Section III gives an overview about the calculation of induced voltages while Section IV explains how the transmission BERs are calculated. Section V highlights the three major insights obtained from the study of time diversity for multi-frequency disturbances and Section VI lists some concluding remarks.

\section{Problem Formulation}

The analysis was carried out using an in-house built simulation framework in which the geometry, encoding scheme, EMI environment and the voting scheme can be freely selected. The following sections give an overview of the simulation set-up.

\section{A. Geometry Under Study}

The simulated geometry is shown in Fig 1 . It consists of three redundant channels parallel to each other with equal spacing. The same geometry is chosen as in [5] where time diversity was studied for single-frequency EMI. The three parallel micro-strips are driven by $1 \mathrm{~V}$ power supply with a source impedance of $50 \Omega$ and are terminated with a $50 \Omega$ load impedance.

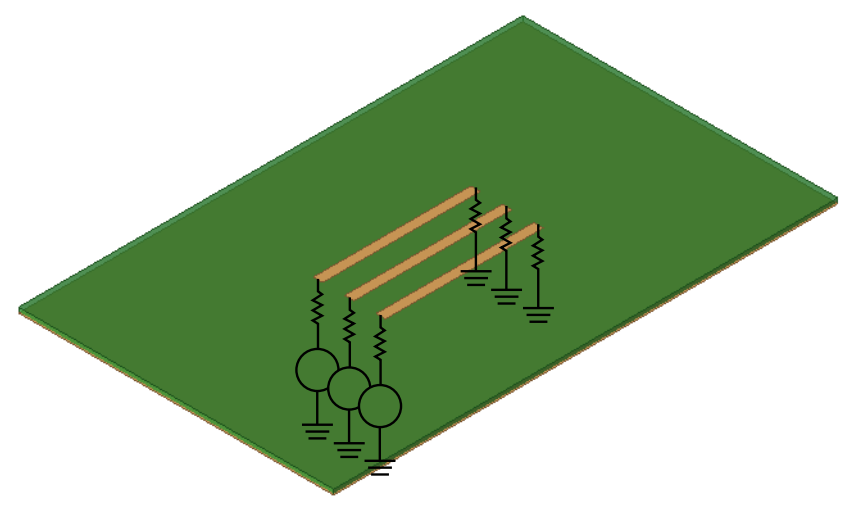

Fig. 1. Three channel redundant system

Since the trace's characteristic impedance is matched to the load at the output port, the maximum output voltage for a $1 \mathrm{~V}$ applied signal is equal to $0.5 \mathrm{~V}$. The same bitstream is transmitted over the redundant channels as shown in Fig. 1 while the geometry at the same time is disturbed by EMI.

\section{B. Encoding Scheme}

A random stream of 100 bits is first encoded into voltages using a Non-Return-to-Zero-Level (NRZ-L) encoding scheme. A ' 0 ' bit is encoded as $0 \mathrm{~V}$ while a ' 1 ' is encoded as $1 \mathrm{~V}$. EMI disturbance for each statistical angle of incidence is sampled at the middle of each bit period. Fig. 2 shows a snapshot of a $200 \mathrm{MHz}$ EMI disturbance sampled on a 200

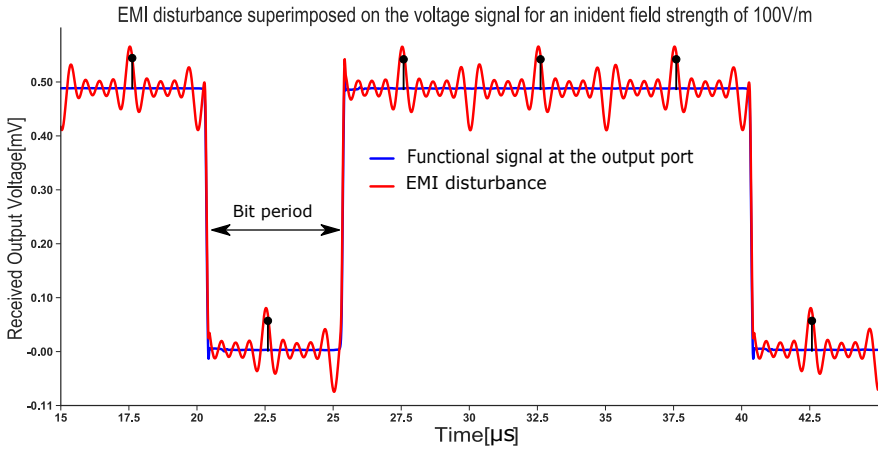

Fig. 2. EMI sampling at the middle of each transmitted bit

MHz encoded bit stream.

\section{The Idea behind Time Diversity}

The fact that EMI leads to a common cause failure, meaning that identical components are likely to fail in a similar manner, makes redundancy on its own insufficient for improving the overall system's resilience to EMI. Diverse redundant architectures for EMI have been explored using time diversity [5], frequency diversity [11], spatial diversity [6] and different matching schemes [7]. For time diversity, it was shown in [5] that provided that the predominant EMI disturbance frequency is known, a sinewave can always be sampled at its most diverse points for each redundant channel. Then the sampled voltage is as distinct as possible, thereby maximizing the time diversity's effectiveness.

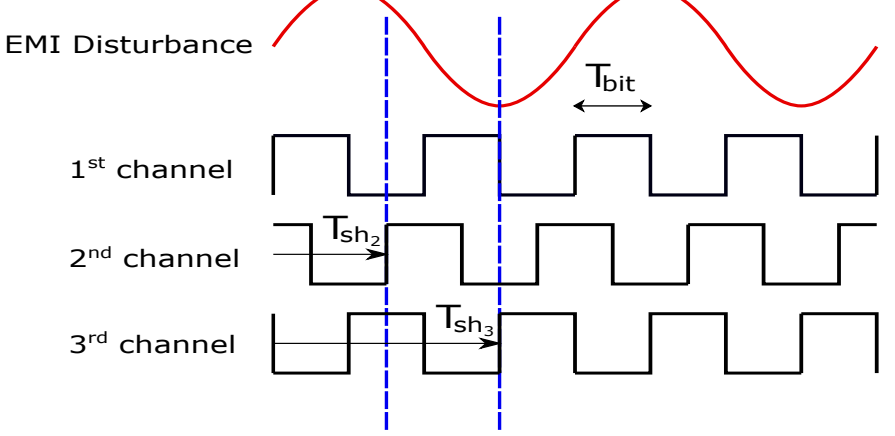

Fig. 3. Concept of time diversity for single frequency EMI disturbance

Fig. 3 explains the concept of time diversity for a single frequency EMI disturbance. The bits transmitted across redundant channels are mutually shifted so that the incoming EMI disturbance is sampled at a different time instance in each channel. This leads to a different voltage being induced in each individual channel. When combined with a suitable voting scheme, this technique can be used as an effective countermeasure against the common cause effects of EMI. Time 
diversity technique for a three channel system is governed by the following relationship.

$$
\begin{aligned}
& T_{S H_{2}}=d_{o} \cdot T_{D I V} \\
& T_{S_{H_{3}}}=d_{1} \cdot T_{D I V}
\end{aligned}
$$

where $T_{D I V}$ is the period of the chosen time diversity frequency, $d_{o}$ and $d_{1}$ are constants, and $T_{S_{H_{2}}}$ and $T_{S_{3}}$ are the bit delays in the $2^{\text {nd }}$ and $3^{\text {rd }}$ channels, respectively.

For optimal time diversity for a single frequency EMI, it was shown in [5] that shifting the bit signal by 120 degrees with respect to the EMI signal from channel to channel leads to the most diverse behavior and, hence, an optimal improvement in terms of the BERs when the traces are close to each other. In other words choosing $T_{D I V}$ equal to the period of the interfering EMI i.e. $T_{E M I}$, and choosing $d_{o}$ and $d_{1}$ as $1 / 3$ and $2 / 3$,respectively leads to the scenario where a sinewave ends up being sampled at its most distinct points. For the ensuing analysis of time diversity for multi-frequency disturbances, $T_{E M I}$ would correspond to the period of the composite EMI disturbance while we keep $d_{o}$ and $d_{1}$ the same as $1 / 3$ and $2 / 3$, respectively.

\section{Voting Scheme}

Redundancy, EM-diversity and majority voting are used to embed fault tolerance in the design. So for a three-channel redundant system a voting based on a '20o3' scheme is used to decide the voltage level at the output ports. For decoding the voltages at the output ports two thresholds are considered i.e. $1 / 3^{\text {rd }}$ and $2 / 3^{\text {rd }}$ of the maximum output voltage. Received voltages below $1 / 3 V_{o}$ are decoded as ' 0 ' and above $2 / 3 V_{o}$ are decoded as ' 1 '. The received voltages in between these two thresholds are decoded as ' 2 ' and are deemed as faulty bits. Fig. 4 explains the decoding procedure used in our simulation model.

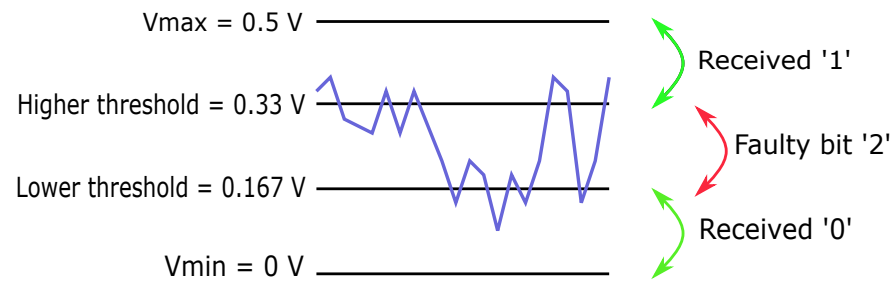

Fig. 4. Decoding of received signal at the output ports

The majority voter makes the final decision about the bit which is actually received by looking at the three received decoded bits. The majority voter's outcome is the value that occurs two or three times among the three received decoded bits. Of course, it can and will happen that the majority voter's outcome is different from the actual transmitted bit. In this case, a bit error occurs as shown in Fig. 5. Finally, note that if any of the three received voltages falls in between both thresholds( the case of faulty bit '2' in Fig. 4), a bit error is always assumed.

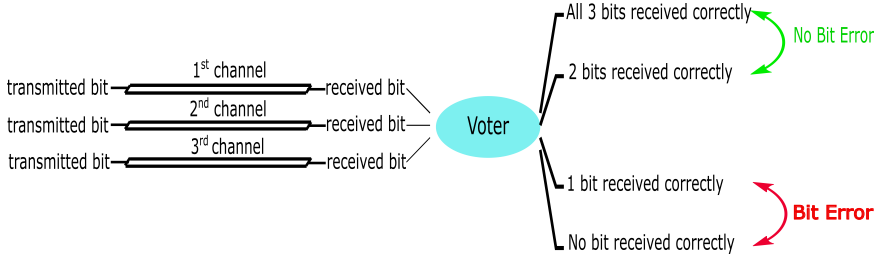

Fig. 5. '20o3' Majority voting scheme

\section{E. EMI Environment}

Simultaneous EMI disturbances at different frequencies can occur in real-life scenarios and thus any EMI-resilient technique ought to be tested in a harsh EM environment for its true merit. Hence, the simulated EMI environment in this study is modified to a square-wave excitation comprising of various harmonics of the fundamental frequency. A previous study [10] has shown that the same three channel communication system becomes significantly more susceptible to multifrequency EMI disturbances and leads to worse performance in terms of the BERs.

To test the susceptibility of the system under test to harmonics of the fundamental frequency, a square waveform is constructed wherein all the harmonic components are in phase as shown in Fig. 6.

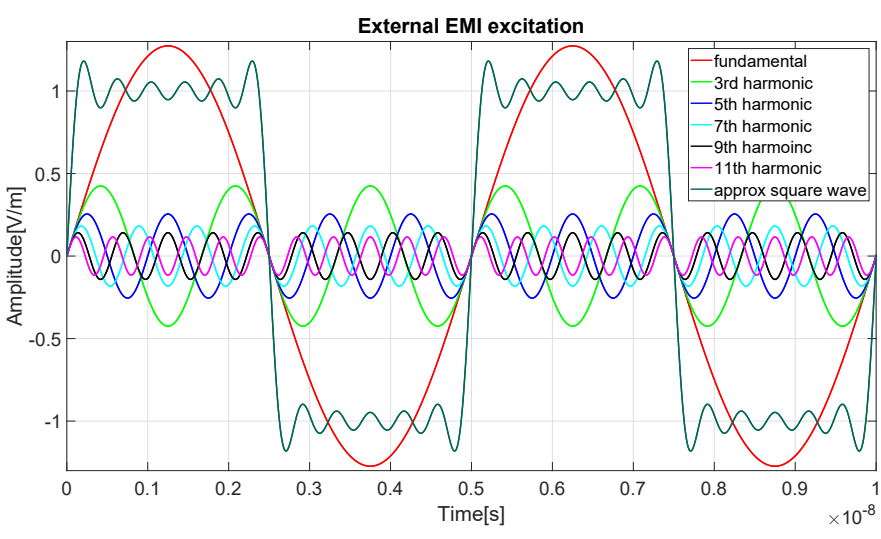

Fig. 6. $200 \mathrm{MHz}$ squarewave EMI excitation along with its harmonic components

\section{Calculation of Induced Voltages}

To calculate the induced voltages on the redundant threechannel system in an efficient manner, the antenna reciprocity theorem is used [8]. Using this algorithm, the geometry's far-field response in the transmit mode is captured for each port and each harmonic frequency with a single full wave simulation. The far-field patterns along with the characteristics of the particular EMI waveform are then used to calculate the received voltage at the ports in the time domain.

As can be seen in Fig. 7, the actual induced voltage at the port terminals is different from the actual impinging external square-wave EMI disturbance. 


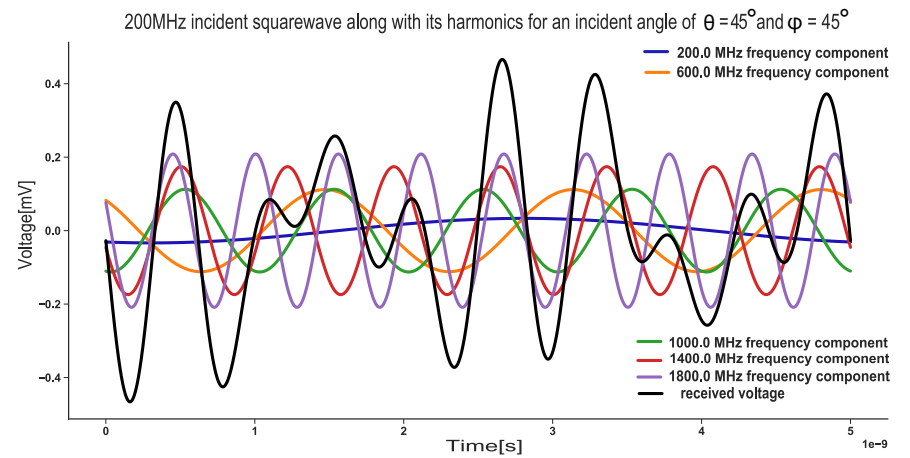

Fig. 7. Received port voltage along with its harmonic frequency components

\section{Bit Error Rate Calculation}

The BER is calculated by sweeping the external multifrequency EMI over all the possible angles of incidence, polarizations and phases with which it can impinge on the device under test (DUT). Fig. 8 shows a time domain visualization of a theta-polarized EMI disturbance for three different angles of incidence.

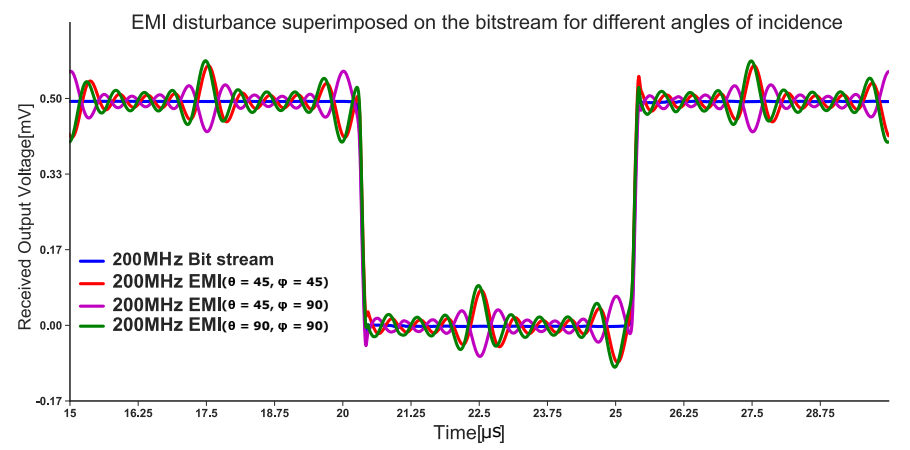

Fig. 8. Time domain visualization of EMI superposition on the transmission signal

The BER is then calculated as the total number of incorrectly received bits for a certain bit pattern for all possible angles of incidence $(\theta, \phi, \psi)$ and time delays $(\alpha)$ with which the square wave disturbance can interfere with the transmitted bit stream. Mathematically the BER can be expressed as

$$
\mathrm{BER}=\frac{\text { number of bits received incorrectly }}{\text { bitpattern length } . n_{\theta s} \cdot n_{\phi s} \cdot n_{\psi s} \cdot n_{\alpha s}}
$$

where

$n_{\theta s}$ represents the number of polar angles.

$n_{\phi s}$ represents the number of azimuthal angles.

$n_{\psi s}$ represents the number of polarization angles.

$n_{\alpha s}$ represents the number of time delays considered.

A uniform distribution of time delays is assumed over the entire period of the disturbance signal. Fig. 9 shows three delayed versions of a $200 \mathrm{MHz}$ square-wave EMI superimposed on the actual bitstream. In our simulations 41 time delays are considered over the range of $0-\pi \mathrm{rad}$.

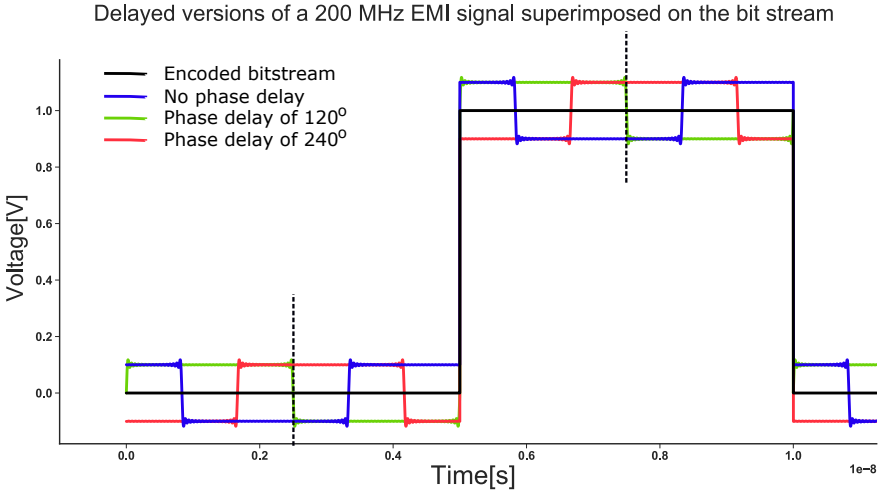

Fig. 9. Uniformly distributed delayed versions of a $200 \mathrm{MHz}$ EMI signal superimposed on the transmitted bit signal

\section{TIME DIVERSITY FOR MULTI-FREQUENCY DISTURBANCES}

To extend the idea of time diversity to multi-frequency disturbances, a simulation was carried out to compute the BERs as a function of the square-wave fundamental frequency. BERs are computed with a resolution of $25 \mathrm{MHz}$ over a fundamental frequency range of 0 to $1 \mathrm{GHz}$ wherein each square-wave contains contributions from its first 5 odd harmonics.

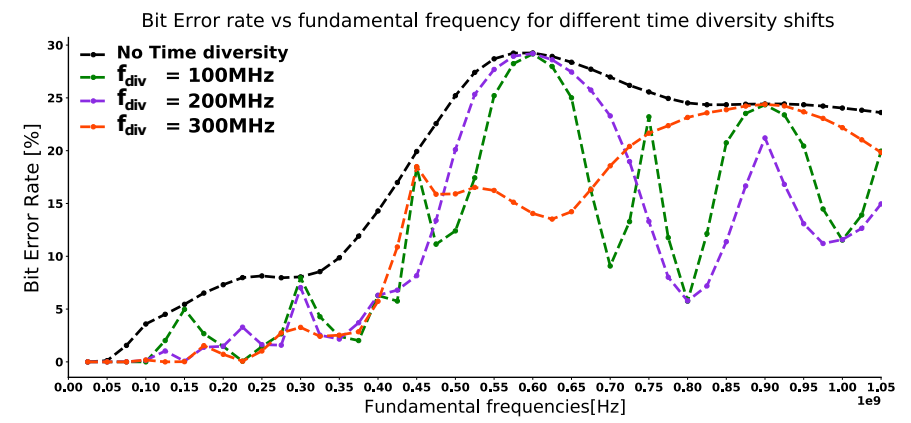

Fig. 10. Comparison of time diversity implementations with the base case

The black curve in Fig. 10 represents the base case where no time diversity is applied. The green, purple and orange curves represent the scenarios where time shifts corresponding to time diversity frequencies of $100 \mathrm{MHz}, 200 \mathrm{MHz}$ and $300 \mathrm{MHz}$ are used. At first glance it appears that the application of time diversity leads to a general improvement in performance over the considered frequency range in terms of the BERs. The following sections give a more detailed analysis of the individual insights that the simulation has revealed.

\section{A. Frequencies With no Improved EM-Diversity}

The initial points worth noticing are the frequencies for which time diversity does not lead to any improvement in terms of the BERs. As can be seen in Fig. 11, at the 'undiverse' frequencies, we witness the same BERs as the base case where no time diversity is applied.

At closer inspection, it becomes apparent that the un-diverse 


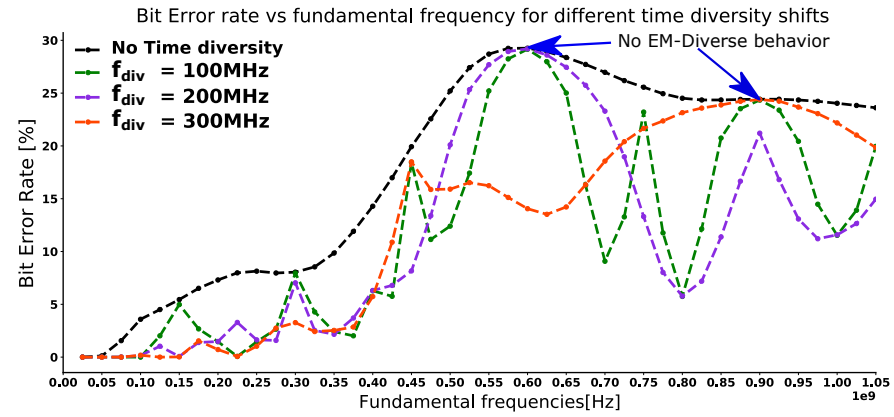

Fig. 11. Frequencies at which time diversity doesn't lead to any improvement in terms of the BERs

frequencies are the ones where the time shifts due to time diversity are a multiple of the $3^{\text {rd }}$ harmonic of the chosen time diversity frequency. For instance, the case of a $600 \mathrm{MHz}$ EMI signal with a time diversity frequency of $100 \mathrm{MHz}$ can be seen in Fig. 12. As $600 \mathrm{MHz}$ is a multiple of the $3^{\text {rd }}$ harmonic of the time diversity frequency of $100 \mathrm{MHz}$, even with time shifts in redundant channels, EMI is getting sampled at the same instance. The voter therefore, perceives the same signal across each channel and thus no improvement in BER is possible.

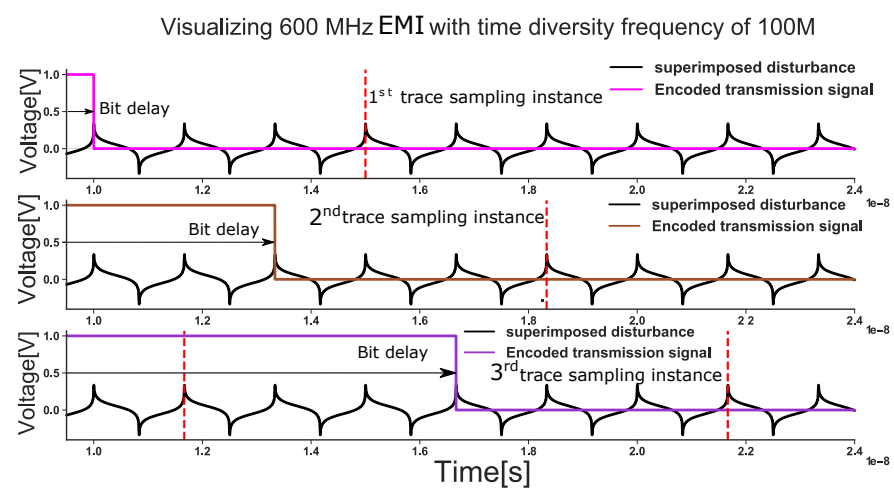

Fig. 12. Sampling instances on the three different channels for the undiverse scenario

\section{B. Frequencies Corresponding to Local Maximas}

Local maximas are the regions which correspond to peaks in the plot with only a minimal improvement in the BER as shown in Fig. 13.

To explain this phenomenon let's take the example of the peak in the green curve for a $1.05 \mathrm{GHz}$ EMI signal with a time diversity frequency of $100 \mathrm{MHz}$. Applying optimal diversity formulas (1), (2), the time shifts for each channel can be calculated as

$$
\begin{gathered}
T_{S H_{2}}=\frac{T_{D I V}}{3} \Rightarrow 3.3 \mathrm{~ns} \\
T_{S H_{3}}=\frac{2 T_{D I V}}{3} \Rightarrow 6.6 \mathrm{~ns} \\
f_{E M I}=1.05 \mathrm{GHz} \Rightarrow T_{E M I}=0.95 \mathrm{~ns} \\
n=\frac{T_{S H_{1}}}{T_{E M I}} \approx 3.5
\end{gathered}
$$

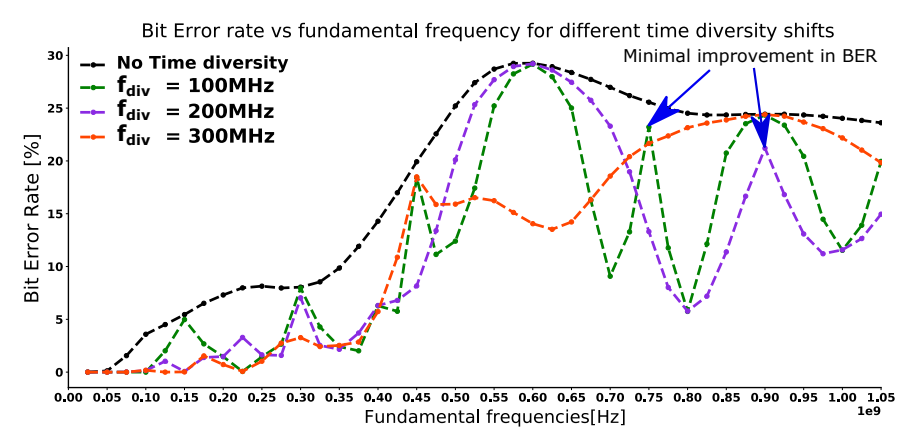

Fig. 13. Maxima points with minimal improvement in terms of the BERs

where $n$ is the ratio of the applied time shift and the EMI time period. Fig. 14 shows a visual illustration of the case under study. The voltage superimposed on the $2^{\text {nd }}$ trace is $3.5 \mathrm{EMI}$ periods delayed in comparison to the signal sampled on the $1^{\text {st }}$ trace and the $3^{\text {rd }}$ trace in turn samples a voltage which is another 3.5 EMI periods delayed. As a result of $n$ being a half-integer the $2^{\text {nd }}$ trace samples a voltage which is $180^{\circ}$ delayed to the original EMI signal while the $3^{\text {rd }}$ trace samples the same voltage as that on the $1^{\text {st }}$ trace.

\section{Visualizing $1.05 \mathrm{GHz}$ EMI with time diversity frequency of $100 \mathrm{M}$}

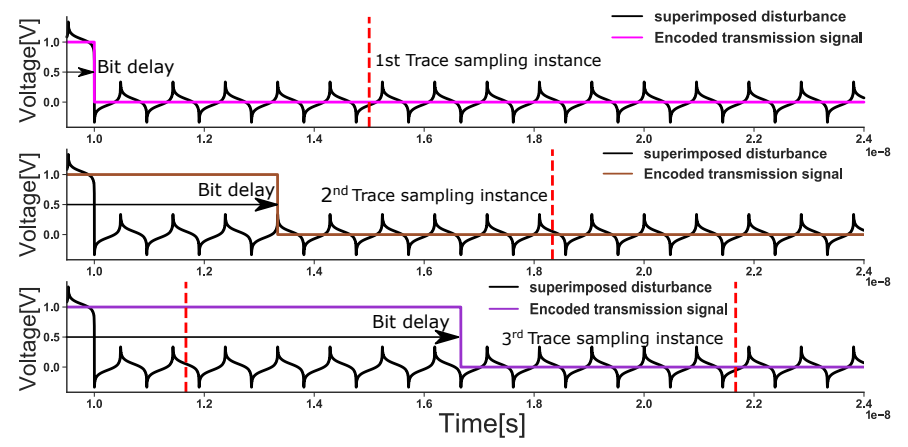

Fig. 14. Sampling instances on the three different channels for points corresponding to maximas

If $n=\mathbb{Z}+\frac{1}{2}$ for the set of all integers $\mathbb{Z}$ then mutual time shifts of $180^{\circ}$ would cause two of the three channels to sample the same signal. Hence, in this case of a three channel redundant system, the 2003 voter would not be able to recover the double fault scenario and, as a result, we see only a slight improvement in the BERs.

\section{Frequencies Corresponding to Local Minimas}

Frequencies correponding to the local minimas are the ones where a substantial improvement in terms of the BERs is seen. In other words, these are the regions where time diversity performs the best. Let's take the example of a $800 \mathrm{MHz}$ EMI disturbance with a time diversity frequency of $200 \mathrm{MHz}$ which represents a local minimum in Fig. 15.

Using the same analysis as in section VI.B we can calculate $n$.

$$
T_{S H_{2}}=\frac{T_{D I V}}{3} \Rightarrow 1.66 \mathrm{~ns}
$$




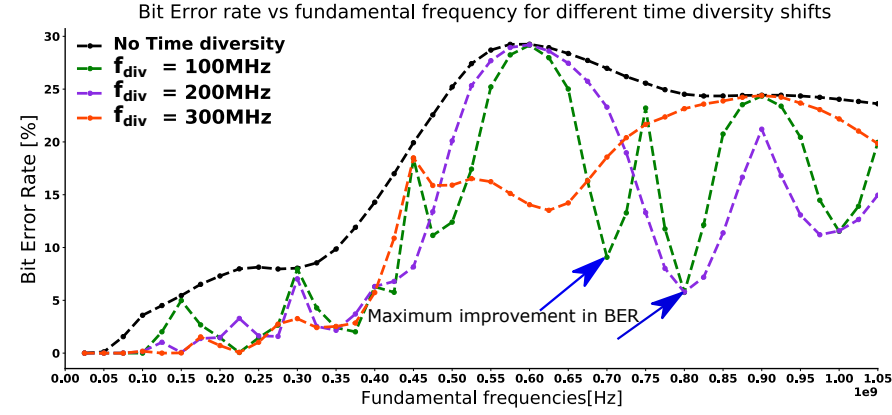

Fig. 15. Minima points with a substantial improvement in terms of the BERs

$$
\begin{gathered}
T_{S H_{3}}=\frac{2 T_{D I V}}{3} \Rightarrow 3.33 \mathrm{~ns} \\
f_{E M I}=800 \mathrm{MHz} \Rightarrow T_{E M I}=1.25 \mathrm{~ns} \\
n=\frac{T_{S H}}{T_{E M I}} \approx 1.33
\end{gathered}
$$

Fig. 16 shows a visual representation of the case under study. The sampling instances for the $2^{\text {nd }}$ and the $3^{\text {rd }}$ traces are shifted by approximately $4 / 3^{\text {rds }}$ and $8 / 3^{\text {rds }}$ of the EMI period, respectively. Since we are dealing with a three-channel system, mutual time shifts of $120^{\circ}$ with respect to the fundamental EMI period lead to the most diverse situation and, hence, the lowest BERs.

Visualizing $800 \mathrm{MHz}$ signal with time diversity frequency of $200 \mathrm{M}$

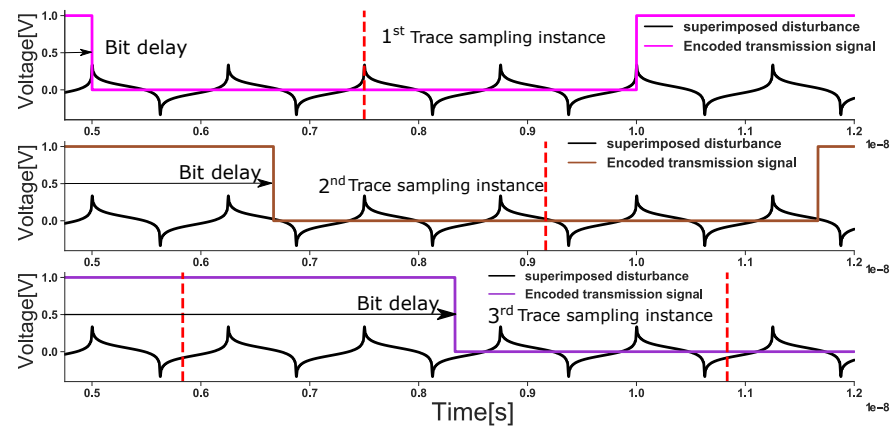

Fig. 16. Sampling instances on the three different channels for points corresponding to minimas

\section{CONCLUSIONS}

A comprehensive study was undertaken to investigate the merit of time diversity as a viable EMI resilience measure in harsh electromagnetic environments represented by simultaneous multi-frequency disturbances. As a particular case, a square wave excitation comprising of various harmonics was chosen as the source for the electromagnetic disturbance in planewave conditions. Results have shown that time diversity indeed proves to be an effective mitigation measure against EMI because of a demonstrable improvement in terms of the BERs over a large part of the the frequency range of interest. However, the results have also pointed towards certain limitations of the time diversity technique whereby for certain frequencies, the system doesn't behave as EM-diverse. As future work, it would be worth investigating time diversity in combination with other hardware based EM-diversity techniques to further improve fault tolerance capabilities of EMdiverse systems.

\section{REFERENCES}

[1] K. Armstrong, "Why Increasing Immunity Test Levels is not Sufficient for High-Reliability and Critical Equipment," 2009 IEEE International Symposium on Electromagnetic Compatibility, Austin, TX, 2009, pp. 30-35.

[2] D. Pissoort and K. Armstrong, "Why is the IEEE Developing a Standard on Managing Risks due to EM Disturbances?," 2016 IEEE International Symposium on Electromagnetic Compatibility (EMC), Ottawa, ON, 2016, pp. 78-83.

[3] D. Pissoort, J. Lannoo, J. V. Waes, A. Degraeve and J. Boydens, "Techniques and Measures to achieve EMI Resilience in Missionor Safety-Critical Systems," in IEEE Electromagnetic Compatibility Magazine, vol. 6, no. 4, pp. 107-114, Fourth Quarter 2017.

[4] K. Armstrong, "Techniques and Measures to Manage Functional Safety and Other Risks with Regard to Electromagnetic Disturbances," 2018 IEEE 4th Global Electromagnetic Compatibility Conference (GEMCCON), Stellenbosch, South Africa, 2018, pp. 1-5.

[5] J. Lannoo, J. Van Waes, A. Degraeve, D. Vanoost, J. Boydens and D. Pissoort, "Effectiveness of Time Diversity to Obtain EMI-Diverse Redundant Systems," 2018 International Symposium on Electromagnetic Compatibility (EMC EUROPE), Amsterdam, 2018, pp. 288-292.

[6] A. Degraeve and D. Pissoort, "Study of the Effectiveness of Spatially EM-Diverse Redundant Systems Under Reverberation Room Conditions," 2016 IEEE International Symposium on Electromagnetic Compatibility (EMC), Ottawa, ON, 2016, pp. 374-378.

[7] J. Lannoo, A. Degraeve, D. Vanoost, J. Boydens and D. Pissoort, "Study on the Use of Different Transmission Line Termination Strategies to Obtain EMI-Diverse Redundant Systems," 2018 IEEE International Symposium on Electromagnetic Compatibility and 2018 IEEE AsiaPacific Symposium on Electromagnetic Compatibility (EMC/APEMC), Singapore, 2018, pp. 210-215.

[8] F. Vanhee, D. Pissoort, J. Catrysse, G. A. E. Vandenbosch and G. G. E. Gielen, "Efficient Reciprocity-Based Algorithm to Predict Worst Case Induced Disturbances on Multiconductor Transmission Lines due to Incoming Plane Waves," in IEEE Transactions on Electromagnetic Compatibility, vol. 55, no. 1, pp. 208-216, Feb. 2013.

[9] Hill, David A. "Plane Wave Integral Representation for Fields in Reverberation Chambers." IEEE Transactions on Electromagnetic Compatibility 40.3 (1998): 209-217.

[10] H. Tirmizi, J. Lannoo, D. Vanoost, G.A.E Vandenbosch, D. Pissoort. "Effects of an External Multi-harmonic EMI Excitation on the Transmission Bit Error Rates of a Redundant Channel under Plane Wave Illumination" accepted for EMC Europe, 2020.

[11] J. Lannoo, J. Van Waes, A. Degraeve, D. Vanoost, J. Boydens and D. Pissoort, "Effectiveness of Frequency Diversity to Obtain EMIDiverse Redundant Systems," submitted to the IEEE Transactions on Electromagnetic Compatibility. 